How to cite: Moga, I.C., Petrescu, G., Doroftei, B.I., Buzea, R. (2019) Water Management Applied in Recirculating Aquaculture Systems. 2019 "Air and Water - Components of the Environment" Conference Proceedings, ClujNapoca, Romania, p. 357-364, DOI: 10.24193/AWC2019_35.

\title{
WATER MANAGEMENT APPLIED IN RECIRCULATING AQUACULTURE SYSTEMS
}

\author{
Ioana Corina MOGA ${ }^{I_{凶}, \text { Gabriel PETRESCU }}{ }^{1}$, Bogdan Iulian DOROFTEI', \\ Roxana BUZEA ${ }^{I}$
}

DOI: 10.24193/AWC2019 35

\begin{abstract}
The authors present results and an improved wastewater technology applied in recirculated aquaculture systems. Due to the high value of the investment, the total number of the recirculated aquaculture systems is small compared to the classical (cages, flow-through, ponds etc.) aquaculture. The high investment cost is given by the existence of the wastewater treatment stage. That is why, new cost-effective technologies for wastewater treatment are developed. The authors propose an improved mobile bed biofilm reactor to be used within recirculated aquaculture systems. The nitrogen compounds (ammonia - the main contaminant produced by the fish metabolism, nitrates and nitrites) are thought to be the main pollutants generated by the recirculating aquaculture systems. The improved technology was tested in laboratory conditions and the obtained results confirm the improvement of the treatment efficiency. A new (material and shape) biofilm carrier, used in mobile bed biofilm reactors, is described by the authors. The new material has led to an increased quantity of the attached microorganisms by more than $250 \%$ compared to the plastic materials (high density polyethylene) commonly used for the biofilm carrier manufacture. Synthetic wastewater was used during the experiments.
\end{abstract}

Keywords (4-6): wastewater treatment, recirculated aquaculture systems, mobile bed biofilm reactor, polyethylene, Romania

\section{INTRODUCTION}

During the last decades, the recirculating aquaculture systems (RAS) become more attractive compared to the classical fish farmers. Within RASs, approx. $90 \%$ of the wastewater generated by the fishes is recirculated and treated in a wastewater treatment plant (WWTP) and recirculated, the rest of approx. 10\% being fresh water. Regarding the wastewater treatment within RAS the main problems are caused by:

- suspended solids (SS) that result from food leftovers and fish faeces. The suspended solids may cause severe problems within RAS - it can limit the amount of fish that can be reared into the system and may cause irritation to the fishes' gills. Due to their origin, the suspended solids are mainly composed of organic matter which can quickly led to putrescence and decrees the concentration of the dissolved

${ }^{1}$ DFR Systems SRL, Drumul Taberei 46, 061392, Bucharest, Romania, corinamoga@yahoo.com dfr@dfr.ro 
oxygen. The majority of SS are removed by mechanical filtration such as settlers, filters (drum, sand) and the rest are reduced in the biological treatment stage.

- microorganisms that can cause diseases. Because the water within RAS is recirculated, disinfection without chemicals is recommended. The ultraviolet light and/or ozonation are commonly used.

- the nitrogen compounds (ammonia, nitrites and nitrates). These compounds are considered the main contaminants in the RAS wastewater. Ammonia is the main waste produced by the metabolism of fish. In order to reduce / eliminate harmful waste products, biological treatment stages are introduced in the WWTPs.

- carbon dioxide. Currently the carbon dioxide is removed from water by using aeration techniques or striping.

- maintaining a certain level of water temperature.

The authors' researches are related to the decrease of the total organic matter and of the nitrogen compounds based on the biological treatment within a WWTPs. The biological treatment that uses biofilm carriers has as a basic principle the development and fixation of an important population of microorganisms on an artificial mobile support. In the specialty literature, this treatment process is found under the name Moving Bed Biofilm Reactor (MBBR) and first was developed in Norway (Ødegaard et al., 2013). The biofilm carriers have a large exposure area for biofilm fixation and that is why the treatment process is faster compared to the classic active sludge treatment. The soil footprint of MBBR-type treatment plants is greatly reduced because in such systems the amount of biofilm developed per volume unit is much superior to the soil footprint of an activated sludge WWTP. Further improvement of MBBR requires development on new biofilm carriers capable of sustaining an increased number of microorganisms and selection of consortia of microorganisms capable of more efficient pollutant removal.

MBBRs are mainstream in water treatment (Torresi et al., 2016). The microorganisms inside tanks form biofilms, that attach to small pieces immersed in wastewater, also known as biomedia or biofilm carriers (Levsteck and Plazi, 2009). Most commonly, the biofilm carriers are realised from plastic materials such as highdensity polyethylene (HDPE) or polyvinyl alcohol (PVA) (Barwal and Chaudhary, 2014). Wastewater flows through the biological treatment reactors and the attached biofilms eliminates/reduces the pollutants. Most of the artificial mobile support is made of plastic materials with density close to $1 \mathrm{~g} / \mathrm{cm}^{3}$. Having a close-to-water density is insured an efficient mixing inside the tanks. The biofilm carriers' utilization is efficient in removal of different pollutants (Pham et al., 2008). Inside a MBBR, in aerobic conditions, several oxidation reactions occur, and different type of pollutants are reduced: ammonia/nitrite, organic carbon, sulphur, heavy metals. The efficiency of nitrogen compounds removal in bioreactors was studied extensively (Picurell et al., 2016). Researches have proven that many factors (biofilms' thickness, wastewater flow-rate, quantity of ammonia and dissolved oxygen) affect the nitrification efficiency (Torresi et al., 2016). Other researches (Hu et al., 2009) revealed a negative correlation between the amount of dissolved organic carbon and the nitrification efficiency. 
Some of the authors developed 2 two biofilm carriers to be used inside MBBRs (Patent RO123174/2008 and Patent request A/01053/2017). By using the patented biofilm carrier, the authors have obtained the following results relative to activated sludge methods: more efficient nitrification; higher efficiency of dissolved organic compound removal; small bioreactors' footprint; capacity to treat high loaded wastewaters within small tanks.

The main objective of the present study is to reveal the efficiency of the innovative biofilm carriers inside a RAS. Several experiments on a laboratory RAS were realized.

\section{DATA AND METHODS}

\subsection{Data used}

\subsubsection{Synthetic wastewater used for experiments}

The receipt for the synthetic wastewater used during the experiments was selected from the literature and realized by the Institute of Biology Bucharest and was based on a mixture between different components of which: peptone, urea, bouillon extract etc. The authors performed the analysis of the synthetic wastewater and the main pollutants have the following concentration: $\mathrm{NH}_{4}-4.43 \mathrm{mg} / \mathrm{L} ; \mathrm{NH}_{3}-4.18 \mathrm{mg} / \mathrm{L}$; $\mathrm{NO}_{3}{ }^{-}-571.9 \mathrm{mg} / \mathrm{L} ; \mathrm{NO}_{2}{ }^{-}-1.15 \mathrm{mg} / \mathrm{L} ; \mathrm{CBO}_{5}-240.50 \mathrm{mg} / \mathrm{L} ; \mathrm{TOC}-129.06 \mathrm{mg} / \mathrm{L}$.

\subsubsection{Materials used for biomedia realization}

Two material were used for the biomedia realization namely high density poliethylene (HDPE) and a mixture between HDPE and an anorganic material (this receipt will be the subject of another patent request).

\subsection{Methods}

The authors conceived an innovative laboratory installation for the determination of the wastewater treatment efficiency within a recirculated aquaculture system. The laboratory installation was conceived to test 4 different biological wastewater treatment technologies and to choose the most efficient process to be further developed.

Laboratory installation has one principal tank where the synthetic wastewaters may be introduced. From this principal tank, the wastewater is directed into the 4 treatment lines, as is presented in Fig. 1.

These 4 lines have the same flow-rate. In this way, the 4 biological treatment stages will have the same inflow (the same: quantity of wastewater and pollutants; temperature).

The four biological lines are: Line 1 - biological treatment with biofilm carriers; Line 2 - biological treatment with algae and biofilm carriers; Line 3 - biological treatment with algae; Line 4 - biological treatment based on Lemna sp. and algae. As previous mentioned, 2 types of biofilm carriers (Fig. 2) were used during the experiments.

Also, the 2 biofilm carriers were compared from the point of view of biofilm attachment. These determinations were made under the guidance of the researchers from the Institute of Biology Bucharest, by the direct coordination of prof. Ioan Ardelean. 


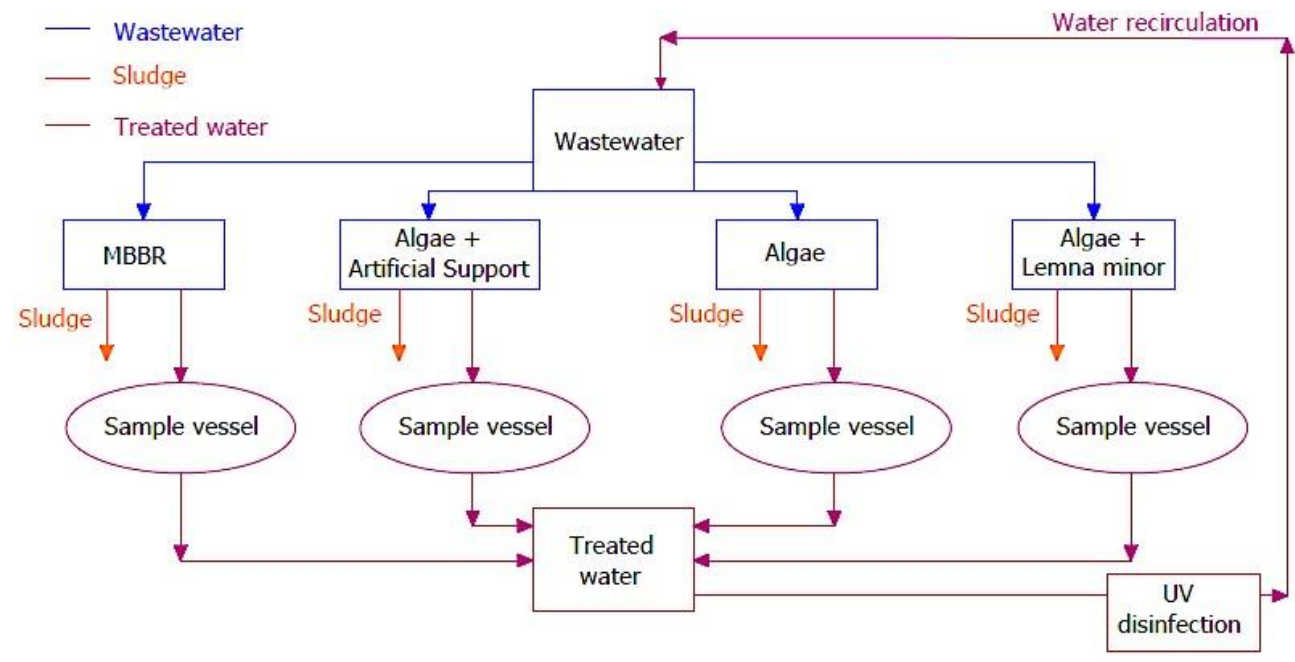

Fig. 1. Technological flux of the laboratory installation used to determine the efficiency of different biological wastewater treatment processes inside RASs

Also, the infrastructure of the Institute of Biology Bucharest was used. The methodology used for the determinations is based on the utilization of crystal violet solution (O'Toole, 2011).
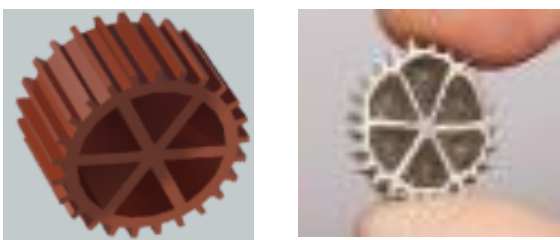

a. Biofilm carrier according to the Patent RO123174/2008

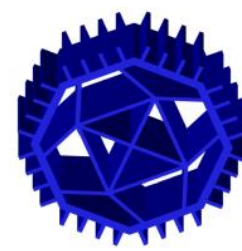

b. Biofilm carrier according to the Patent request $\mathrm{A} / 01053 / 2017$

Fig. 2. Biofilm carriers used for the present experiments.

Three main stages were followed during the experiments: biofilm formation on the biomedia (the biofilm carriers were introduced in synthetic wastewater and selected microorganisms were added by the Institute of Biology Bucharest); the formed biofilm staining; the determination of biofilm quantity.

\section{RESULTS AND DISCUSSIONS}

The laboratory installation was conceived, designed (Fig. 3) and realized. All the four biological lines were tested, and the MBBR (Fig. 4) treatment provided very good results compared to the other biological processes. 
The main water quality indicators (for both MBBR and synthetic wastewater) were determined in time and compared. Their evolution is presented in Figure 5, where with "_SW" are mentioned the values obtained for synthetic water.

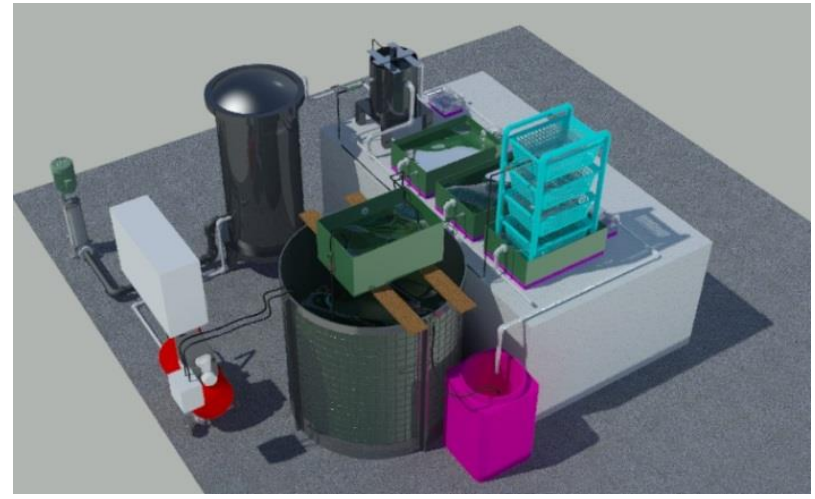

Fig. 3. Laboratory installation

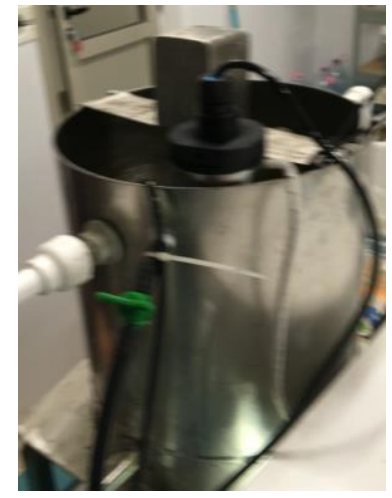

Fig. 4. MBBR tank

By removing the chemical bonds from the different components (meat extract, peptones and urea), of the synthetic wastewater the values of the wastewater quality indicators initially increased, but as a result of the biological treatment process they were again reduced. In this way, the efficiency of the MBBR is proved.

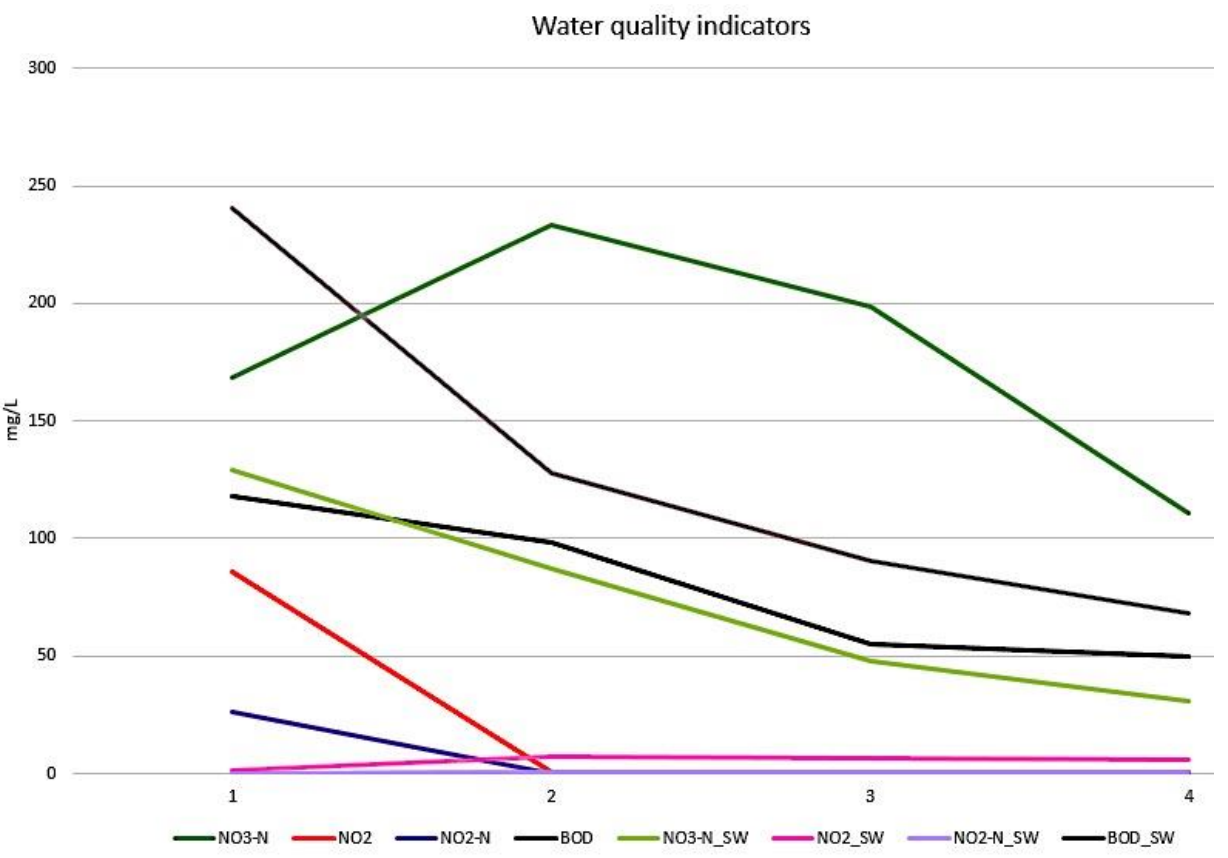

Fig. 5. Values for the main water quality indicators obtained during the experiments 


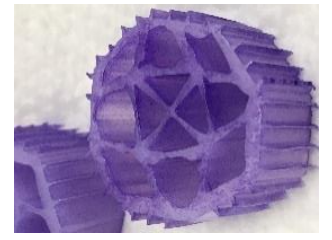

Fig. 6. Biofilm carrier during the experiments with crystal violet
Also, the biofilm adhesion of the two different materials was investigated (Figure 6). Biofilm adhesion to the new material has increased considerably by more than $200 \%$ compared to biofilm carriers made exclusively from HDPE. It is known that HDPE has hydrophobic properties, but the mixture of HDPE with an inorganic material that has hydrophilic properties led to better adhesion of the biofilms.

\section{CONCLUSIONS}

Beside the proved efficiency in wastewater treatment, the new biofilm carriers (Patent request A/01053/2017) proved to be cheaper, up to 25\% per $\mathrm{m}^{3}$, than the one previous utilized (Patent RO123174/2008). In this context, the authors recommend the MBBR utilization in RASs because it meets the requirements of an efficient and cost-effective technology. Innovations in MBBRs are still possible and the authors will try to further develop this biological wastewater treatment process.

As further development of this technology, the researches intend to test the improved biofilm carriers inside anoxic tanks and to test the treatment technology with real wastewaters generated by different species of fish.

\section{ACKNOWLEDGEMENTS}

This work was supported by a grant of the Romanian National Authority for Scientific Research and Innovation, CCCDI - UEFISCDI, project number COFUND-WATER WORKS ERANET 2015-ABAWARE (1), within PNCDI III.

\section{REFERENCES}

1. Barwal A., Chaudhary R. (2014), To study the performance of biocarriers in moving bed biofilm reactor (MBBR) technology and kinetics of biofilm for retrofitting the existing aerobic treatment systems: a review. Reviews in Environmental Science and Bio/Technology, 13(3), 285-299. DOI: https://doi.org/10.1177/0042098017749176

2. Hu J., et al., (2009), Effect of organic carbon on nitrification efficiency and community composition of nitrifying biofilms, Journal of environmental sciences, 3, 387-394. DOI: http://doi.org/10.1016/S1001-0742(08)62281-0

3. Levstek M., Plazi I. (2009), Influence of carrier type on nitrification in the movingbed biofilm process, Water Science \& Technology 59(5), 875-882. DOI: https://doi.org/10.2166/wst.2009.037

4. Moga I.C., Petrescu G, Patent request no. RO CBI A/01052/07.12.2017

5. Nasarimba-Grecescu D.B, Petrescu G., Patent RO123174/2008 
6. Ødegaard, H. (2013), State of the art of wastewater management in the Nordic countries, NORDIWA Conference, 8-10 October, Malmo, Sweden.

7. O'Toole G.A. (2011), Microtiter Dish Biofilm Formation Assay. JoVE. 47. http://www.jove.com/details.php?id=2437, doi: 10.3791/2437

8. Pham H., Viswananthan S., Kelly R.F. (2008), Evaluation of plastic carrier media impact on oxygen transfer efficiency with coarse and fine-bubble diffusers. Proceedings of the Water Environment Federation Technical Exhibition and Conference, Chicago, IL. DOI: http://doi.org/10.2175/193864708788805378

9. Piculell M., Welander P., Jönsson K., Welander T. (2016), Evaluating the effect of biofilm thickness on nitrification in moving bed biofilm reactors, Environmental Technology, 37(6), 732-743. DOI: https://doi.org/10.1080/09593330.2015.1080308

10. Torresi E., Fowler J.S., Polesel F., Bester K., Andersen H.R, Barth F.S., Plósz B.G., Christensson M. (2016), Biofilm Thickness Influences Biodiversity in Nitrifying MBBRs - Implications on Micropollutant Removal, Environ. Sci. Technol., 50(17), 9279-9288. DOI: http://doi.org/10.1021/acs.est.6b02007 
